\title{
Thought Experiments Without Possible Worlds
}

The method of thought experiments or possible cases is widespread in philosophy and elsewhere. Thought experiments come with variegated theoretical commitments. These commitments are risky. They may turn out to be false or at least controversial. Other things being equal, it seems preferable to do with minimal commitments. I explore exemplary ways of minimising commitments, focusing on modal ones. There is a near-consensus to treat the scenarios considered in thought experiments as metaphysical possibilities (most aptly treated as possible worlds). I challenge this consensus. Paradigmatic thought experiments do not have to come with a commitment to metaphysical possibilities. In the first section, I point out difficulties with the prevailing focus on metaphysical possibilities. In the second section, I present alternative formalisations of a paradigmatic thought experiment, the Gettier experiment. Gettier's words leave open the kind of possibilities under consideration. The standard way of spelling out Gettier's argument uses metaphysical possibilities. One alternative proposal uses nomological possibilities. A second one uses epistemic possibilities. My modest conclusion: as long as it is not established that a thought experiment requires a commitment to metaphysical modality, one should avoid such a commitment. My preferred way of doing so is to replace the commitment to one particular formalisation by a commitment to a disjunction of alternative formalisations.

I dwell on possibilities...

Emily Dickinson 
Thought experiments play an important role in philosophy. Even if one cannot do without them, it seems a worthwhile endeavour to explore the minimum commitments incurred in using them. Otherwise one might miss their full dialectical potential. One's argument might fail due to an unnecessary commitment. To avoid this, one should be flexible and attentive to the specific commitments of the particular cases and the dialectical situation. While my caveat concerns any commitment that may be contested, ontological, logical, epistemological or whatever, I shall focus on the kind of modal claim one is committed to in one paradigm case of thought experimenting. Other kinds of commitment and other thought experiments may have to be treated differently. I offer no general criterion of strength of commitment, but I shall rely on the idea that a commitment to the disjunction of $p$ and $q$ is usually weaker than a commitment to $p$. I presuppose an intuitive understanding of metaphysical modality as possibility and necessity according to some absolute standard (though perhaps limited by contextual accessibility). If such an intuitive understanding is doubted, a commitment to metaphysical modality seems even more problematic.

\section{Difficulties with Metaphysical Possibilities}

In the present debate, most philosophers construe thought experimental situations as metaphysical possibilities. I shall challenge this consensus. My aims are modest. I do not advocate modal scepticism, but just argue that paradigm thought experiments better had not come with a commitment to metaphysical necessity or possibility.

To set the stage, I shall present five concerns about such a commitment:

(i) Metaphysical scepticism about metaphysical modalities: one may doubt that there are significant modal truths.

(ii) Epistemic scepticism about metaphysical modalities: even if there are modal truths, one may not be in a position to know them. 
(iii) Exegetical qualms: many thought experiments have not been presented in outright modal language.

(iv) Problems with the incompleteness of descriptions: deviant possibilities.

(v) The problem of missing the target: a counterexample which is presented as a metaphysical possibility can be neutralised by weakening the target claim against which the thought experiment is directed.

These five points deserve to be discussed in depth. Yet I shall use them only as a quick motivation.

(i) In the current debate, few people doubt that there are true modal statements like 'it is (metaphysically) necessary for gold to have atom number 79'. But should we take reasoning in terms of metaphysical modalities for granted? Perhaps we tend to take it for granted only because we are so deeply enmeshed in

an environment composed of philosophers who unthinkingly make all sorts of fanciful modal judgments because they've always been surrounded by philosophers who unthinkingly make the same sorts of fanciful modal judgments.(van Inwagen 1998, 73) ${ }^{1}$

Commitments to metaphysical modalities may arise within everyday or scientific contexts.

Concerning the first ones, when we talk about ways things could or could not be, we normally have in mind contextually restricted situations. Things could be or could not be a certain way given certain circumstances. It does not trivially follow that there is some absolute standard of modality. Even if everyday talk displays a high degree of modal involvement, there are deflationary strategies (cf. O’Leary-Hawthorne 1996, 195). Often one may replace modal talk by talk about categorical properties of things. For instance, when I say that the sofa cannot be moved through the doorway, one may convey the same by saying something about structural properties of the sofa and

$1 \quad$ Van Inwagen himself is moved by epistemological concerns. 
the doorway.

As for scientific contexts, a commitment to metaphysical modality may upon ideal reflection provide the best systematic framework for talking of dispositions, natural laws and the like. Still the framework is likely to come with certain costs, and it is not yet clear that they are outweighed by the benefits.

Considering the costs, modal talk in semantics and metaphysics has not always been as readily accepted as today. Quine famously expressed concerns about losing extensionality (Quine 1953). The possible worlds-framework has been developed to deal with them. But it comes with costs of its own. Few would buy Lewis's (1986a) outright realism about possible worlds. There are attempts at avoiding realism like fictionalism or ersatzism. But these attempts pose new problems (Rosen 1993). Even if the ontological burden can be minimised, there are other concerns about theoretical economy (Huemer 2009). There may be alternatives to metaphysical modality which outdo the possible worlds-framework in theoretical virtues like simplicity, fruitfulness and so on.

In sum, perhaps the abundance of metaphysical modalities is just a philosophical fashion which will be overcome sooner or later without loss in insight. In any case, to weigh the costs and benefits of commitments to metaphysical modality, it seems worthwhile to inquire how far one could get without such commitments.

(ii) Claims to metaphysical possibility or necessity may well be true and play a significant role in an ideal metaphysical theory; still there are doubts that one is in a position to know them. Even if one grants knowledge of everyday possibilities, one may be sceptical about more farfetched possibilities like 'there could be a naturally purple cow', being unable to spell out the relevant details of the purported possibilities (van Inwagen 1998, discussion in Geirsson 2005, Hawke 2011). There are arguments that modal knowledge does not relevantly differ from everyday knowledge claims. A prominent strategy is to reduce the epistemology of metaphysical modalities to the epistemology of innocuous everyday counterfactuals (Williamson 2007). But this move has not gone uncontested (e.g. Roca-Royes 2011, Lowe 2012). 
In sum, knowledge of metaphysical modalities should not simply be taken for granted. Even if the epistemological problems can be overcome, the quest for epistemically less demanding accounts of thought experimental reasoning seems motivated, be it only to see what their minimum epistemic requirements are.

(iii) A different argument starts from the exegesis of actual thought experiments, say Gettier's famous counterexamples. As Williamson notes, Gettier did not use explicit modal talk:

Gettier presents his specific counterexamples to the target analysis through short fictional narratives, in the present tense indicative, with fictional uses of proper names ('Smith' and 'Jones'), all introduced by 'suppose that'.(Williamson 2007, 183)

Indeed Gettier (1963) himself used 'suppose' and 'imagine' + indicative. His supposition may be read as counterfactual. But this is not the literal reading. And though the framework of metaphysical possibilities is standard in treating counterfactuals, there are alternatives. As for 'imagine', many philosophers follow Hume in linking imagination to the appearance of metaphysical possibility (Yablo 1993). But there are many uses of 'imagine' (Langland-Hassan 2014), including one that results in accepting an indicative conditional. Using fictional names does not immediately commit one to metaphysical modality either. Thus, Gettier does not clearly commit himself to metaphysical modalities.

One may present a formalisation in terms of metaphysical modalities as an amendment of Gettier's original reasoning. But many participants in the debate aim at an account of 'our [including Gettier's] actual route' to Gettier's conclusion (Malmgren 2011, 283). No one puts forward an explicitly revisionary account. Thus, one should pay due attention to Gettier's explicit commitments and consider possibilities of a formalisation which stays closer to them.

(iv) Normal descriptions of thought experiments do not explicitly specify every relevant detail. Thus, these descriptions could be realised in a deviant way. The problem has been discussed 
with respect to Gettier stories like the following (taken from Williamson 2009, 467):

$G C$ : At 8:28, somebody looks at a clock to see what time it is. The clock is broken; it stopped exactly twenty-four hours previously. The subject believes, on the basis of the clock's reading, that it is $8: 28$.

The aim of Gettier experiments is to provide a scenario where someone has justified true belief in some proposition without knowing it $(N K J T B)$. Intuitively, $G C$ describes such a scenario. It seems that the subject in the case has NKJTB. But $G C$ is a highly unspecific description, which is true of very different possible scenarios. In many of these scenarios, the subject has NKJTB. But in some of them, the subject does not have $N K J T B$. To obtain such a scenario, just add the following clause to $G C$ :

$G C^{*}$ : ...The subject knew in advance that the clock had stopped exactly twenty-four hours previously.

The clause is perfectly consistent with $G C$. Hence it describes one scenario where $G C$ is satisfied. But the subject in $G C^{*}$, knowing that the clock stopped exactly twenty-four hours earlier, can use the broken clock as an indicator of the time. Intuitively, in doing so, she gets to know the time. Thus we have a realisation of $G C$ where the subject does not have $N K J T B$. This scenario can be called deviant because, though literally satisfying the description of the thought experiment, it does not work as intended by the thought experimenter. For any normal Gettier experiment, there are realisations where the subject knows and realisation where she lacks justification. In order to get a realisation of the latter sort, add the following clause instead of $G C^{*}$ :

$G C^{* *}$ : ...The subject noticed that the second hand was not moving and the glass was 
cracked.

In this case, intuitively, the subject does not have a justified true belief what time it is. She should have taken into consideration salient evidence that the clock was broken.

The resulting problem is the following: the most straightforward formalisation in terms of metaphysical modalities invokes either a strict conditional or a counterfactual. The strict conditional says that any $G C$-scenario is a $N K J T B$-scenario. But the strict conditional is false. Among the $G C$ scenarios in its range, there are deviant $G C$-scenarios as exemplified by $G C^{*}$ (and $G C^{* *}$ ). If the Gettier argument is sound, it cannot depend on the strict conditional as a premiss. The counterfactual says roughly that all the closest $G C$-scenarios are $N K J T B$-scenarios. But the counterfactual is false if one of the closest $G C$-scenarios is deviant as exemplified by $G C^{*}$. By mere coincidence, there might be someone who actually satisfies $G C^{*}$. For the Gettier argument to be sound, we have to assume that there are no closest deviant realisations. But the success of Gettier's experiment does not seem to depend on the assumption that no one actually satisfies $G C^{*}$.

There are alternative formalisations in the literature, which are tailored especially to make the argument deviance-proof (Ichikawa and Jarvis 2009, 2013, Malmgren 2011, Grundmann and Horvath 2014). But arguably our (Gettier's) actual route was not designed to be deviance-proof. We tend to simply ignore deviant realisations and do not safeguard the argument against them. One possible explanation for this tendency is that the spectre of deviant realisations arises only when we think of Gettier scenarios like $G C$ as metaphysical possibilities. Once we construe the Gettier argument in this way, we have to deal with deviant possibilities. But perhaps there are different construals which do not require safeguarding against deviant realisations in the first place.

(v) There is a danger that the evidential significance of a thought experiment is unduly narrowed down if a scenario is presented as a metaphysical possibility. Take a Gettier case which refutes the target theory that knowledge is justified true belief $(J T B)$. The $J T B$-theory may be put as a definition. A definition should, one may say, be conceptually and thus metaphysically necessary. 
Yet Gettier introduces the $J T B$-theory merely as providing necessary and sufficient conditions of knowledge (cf. Williamson 2007, 183). Such conditions may concern only the actual world: In the actual world, any case of $J T B$, past, present, and future, is a case of knowledge and vice versa. If the counterexample is put as a metaphysical possibility, one may rescue the target theory by avoiding a claim to metaphysical necessity. Instead, it may only be claimed that someone has knowledge precisely if she has $J T B$. If this seems too weak, make it a nomological necessity or an a priori claim. In any case, when the thought experimenter comes up with a metaphysical possibility, she may be challenged to show that the $J T B$-theory is committed to denying it. The counterexample mutatis mutandis applies to weaker claims. But the formalisation using metaphysical modalities does not.

\section{Alternative Options}

Construing thought experiments by way of metaphysical modalities gives rise to concerns that might be avoided by alternative construals. I shall explore such alternatives. My prime example being Gettier cases, I start from Williamson's exemplary treatment, which has played a major role in recent debate. In doing so, I focus on certain thought experiments which provide counterexamples to general target claims, leaving open whether other thought experiments require a different construal. While I shall remain modest, I am confident that the model outlined applies more broadly. Though I shall provide straight formalisations, this is not to say that thought experiments can be reduced to them. There is a huge debate on thought experiments in epistemology, metaphysics, and philosophy of science. Due to my limited focus, my argument is neutral with respect to a great many of other relevant issues, for instance whether thought experiments recruit quasi-perceptual rational insights, arguments, imaginations, fictional narratives or whatever. I think that these issues are largely confined to discovering and justifying the premisses used in the formalisations to come, and I shall only occasionally go into questions of discovery and 
justification.

My argument in a nutshell: all we get from Gettier's explicit reasoning (and many similar refuting thought experiments) is a rough-and-ready heuristic; a target claim is confronted with a scenario that somehow conflicts with it. Although the thought experimenter arguably is committed to some precise argument in the neighbourhood, the argument in terms of metaphysical modalities is only one of the alternative precisifications. So all the thought experimenter may be committed to is a disjunction of precise arguments. Only one of them has to be sound. The disjunction may seem convoluted at first glance, but in a concrete debate it boils down to the disjunct which is most eligible. The other alternatives may not even come into view.

Judging from Gettier's explicit text, his reasoning seems guided by some inferential heuristic rather than by rigorous argument. In sketching this heuristic, I do not mean to exhaust what goes on in the thought experimenter's mind. Here is my schema: a target claim is raised which generalises beyond what we so far have found to actually be the case. Therefore it covers a range of cases which do not have to be actual to be relevant. There is a sense in which these cases may be merely possible. Reasoning from such a case to its consequences is done by a supposition ('suppose', 'imagine'). If some consequence is inconsistent with the target claim, the latter is refuted. We should heed the distance between a rough-and-ready argument as presented by Gettier and the formalisations proposed decades later. When I refer to Gettier's original presentation, I have in mind a simplified heuristic like the one outlined above, which I also take to come close to 'our actual route'. It is not to be expected that there is one precise formalisation which perfectly represents our actual route. ${ }^{2}$ Rather there will be several candidates which balance departure from our actual route against other advantages.

There are several ways of making the simple heuristic rigorous. The sense of 'possible' at stake is ambiguous. The ambiguity is usually dissolved in favour of metaphysical modality. All the

2 However, Horvath (2015) tries to develop a pattern of rigorous suppositional reasoning which perfectly captures our actual route and does without metaphysical modality. 
different candidates which follow this line depart more or less from Gettier's original presentation. Closeness to the latter is one virtue that should be balanced against others such as ontological costs, dialectical power and so on. On balance, it is not fully clear that the formalisation by way of metaphysical possibilities prevails. Perhaps the commitment to metaphysical modalities in the Gettier case comes for free because one has to accept such a commitment anyway. But as long as this commitment has not yet been independently established, it may be better to avoid it. One way of avoiding it is to outline several formalisations and to commit oneself only to their disjunction, including those which come with a commitment to metaphysical modality and those which do not.

The freedom of interpreting the heuristic is limited by the dialectical situation. The Gettier experiment may be framed as a cooperative endeavour of succinctly formulating a theory and testing it by crucial cases. The alternatives I present are best interpreted in this way, and the same goes for Gettier's own reasoning. Gettier points to views of Plato, Chisholm and Ayer, but the JTBtheory is his own proposal of what they aim at. Of course the experiment can also be framed as a controversy between proponent and opponent of the $J T B$-theory. In that case, the claim raised by the $J T B$-theorist limits the options of her opponent.

I shall use Williamson's much-debated counterfactual analysis as a point of departure for my alternative formalisations, modifying it in order to avoid the concerns (i)-(v) outlined in the first section (Williamson 2007, 195). 'Is in $G C$ ' abbreviates 'stands to some proposition $p$ in the relation described by $G C^{\prime}$ :

Alternative $A$

(A1) Necessarily, for any subject $S$ and proposition $p, S$ knows $p$ if and only if $S$ has justified true belief in $p$.

(A2) Possibly, some $S$ is in $G C$. 
(A3) If some $S$ were in $G C$, some $S$ would have justified true belief in some $p$ without knowing $p .^{3}$

(A4) It is possible that some $S$ has justified true belief in some $p$ without knowing $p$.

Thus: not (A1)

Williamson's formalisation commits him to metaphysical possibilities. I shall discuss two paradigmatic ways of avoiding this commitment. The first is restricted alethic modality, in particular nomological modality, the second is epistemic modality.

\subsection{Option I: Restricting Alethic Possibilities (Nomological Modality)}

My first option is to restrict one's claims to a smaller and less contentious realm of alethic possibilities than metaphysical ones. This realm should not be arbitrarily confined. Perhaps the most natural restriction of metaphysical possibility beyond actuality is nomological possibility. I shall assume that nomological possibilities are more restricted than metaphysical possibilities (if such there are). I shall start with a basic understanding of nomological possibility: $p$ is nomologically possible precisely if it is not ruled out by the laws of nature. ${ }^{4}$ Concerning the formalisation, we may start with simply reinterpreting the modal operators in Alternative $A$ as nomological possibility and necessity. Call this variant Alternative $B$.

One concern about the counterfactual premiss of Alternative $B$ arises from Lewis's (1986b) semantics for counterfactuals. Simplifying, a counterfactual is true precisely if the closest antecedent worlds are consequent worlds. According to Lewis's criteria for the closest antecedent worlds (deterministic case), a minuscule departure from our actual laws has to bring about the antecedent. As a consequence, Alternative $B$ could not be restricted to nonomogical possibilities. If one endorses Lewis's criteria, I offer two remedies. My preferred suggestion is that the argument in

3 This is already a logically weaker version of Williamson's original proposal (cf. 2007, 199).

$4 \quad$ I am neutral as to whether the laws also include laws of the special sciences. 
terms of nomological possibilities comes with a special context. ${ }^{5}$ The modal horizon is restricted to nomological possibilities. The counterfactual is assessed with respect to the closest nomologically possible worlds. ${ }^{6}$ This move may also allay concerns that the semantics of counterfactuals comes with a commitment to metaphysically possible worlds beyond nomologically possible ones. Lewis presents his criteria as default and hence leaves open the possibility of such a non-default context. The alternative remedy is to vary a strategy developed by Malmgren (2011). She proposes to merge (A2)-(A4) into one a priori claim to possibility:

Possibly, someone is in $G C$ and has NKJTB.

Again the modal operator could be reinterpreted as nomological possibility, skipping the a priori aspect. My concern about a solution along these lines is that the connection between $G C$ and NKJTB is too tenuous. Almost any limited description could somehow describe a case of $N K J T B .^{7}$ A conditional is accepted by most participants in the debate as the most convincing way of expressing the transition from considering $G C$ to taking it to be a case of NKJTB. Hence I offer Malmgren's possibility claim merely as a fallback option. ${ }^{8}$

I shall discuss the pros and cons of Alternative $B$. One advantage is unification. The nomological construal is mandatory for most scientific thought experiments. If it is extended to philosophical thought experiments, the latter can be embedded into a well-established scientific

$5 \quad$ Cf. Smith 2007. His ceteris paribus conditionals might also take care of deviant realisations.

6 It is plausible that the logical principles used by Williamson $(2007,186-187)$ in completing Alternative $A$ apply to Alternative $B$ in this context.

$7 \quad$ For related criticism Ichikawa and Jarvis 2013, 203.

8 An alternative approach is to replace the counterfactual by a suitably restricted strict conditional (cf. Ichikawa and Jarvis 2009, 2013, criticised in section 2.2.1.) 
practice. This is attractive especially for philosophers who bother about philosophical exceptionalism. However, the price of unification is that the proposal does not cover counternomological thought experiments like a human observer traveling at light speed.

How does the strategy fare with the difficulties mentioned in section 1 ?

(i) Metaphysical qualms: Nomological possibilities as introduced have two main advantages. They have been elegantly defined by natural laws. Moreover, we avoid the problematic realm of counternomological possibilities, at least for the Gettier experiment and comparable nomologically possible scenarios. Still there are some metaphysical qualms. The status of laws of nature is a deep metaphysical question. It seems highly likely that laws are independently required, but we may prefer to avoid a special commitment to them in the Gettier experiment and comparable philosophical thought experiments.

A more interesting problem concerns the introduction of nomological possibilities by their compatibility with the laws of nature. I have assumed that nomological possibilities are more restricted than metaphysical possibilities (if such there are). But it is not clear that compatibility with the laws of nature ensures that this assumption is true. Perhaps there are metaphysical necessities which do not follow from the laws of nature. For instance, the laws might be silent about high-level concepts like person and thus leave open whether personal identity over time is necessarily bound to bodily identity. As a consequence, a definition of nomological possibilities only in terms of laws might allow for some $p$ to be nomologically but not metaphysically possible. Perhaps conceptual necessities take care of this problem, but we cannot take this for granted. We may add a proviso: $G C$ is nomologically possible and, provided there are metaphysical possibilities, it is metaphysically possible.

While I cannot discuss these metaphysical concerns and possible reactions to them in depth, I am confident that, on balance, Alternative $B$ remains a metaphysically interesting option for the thought experimenter.

(ii) Epistemological qualms: on the one hand, nomological possibilities seem less far-fetched 
and mysterious than certain metaphysical ones. Moreover, there is a clear pathway to knowing them: do science and figure out the laws. On the other hand, as far as nomological possibilities are more restricted than metaphysical ones, they are subject to additional conditions (fit with the laws). We have to empirically find out whether the additional conditions are satisfied. These requirements often go beyond the empirical knowledge required to settle claims to metaphysical modality. For instance, we need to know whether Gettier scenarios are in tune with the laws. Probably there are easier heuristics to establish the nomological possibility of down-to-earth scenarios like Gettier's, e.g. by analogy with actual scenarios (cf. Hawke 2011), but they may not be available for more farfetched cases. Thus, the epistemological balance of Alternative $B$ is mixed.

(iii) Exegetically, a construal in terms of nomological modalities may be motivated by the tradition of scientific thought experiments, but Gettier's presentation contains no reference to nomological modality. ${ }^{9}$ Thus, Alternative $B$ departs from Gettier's actual route.

(iv) The problem of deviant realisations is mitigated as far as counternomological deviant realisations are concerned, but it remains for nomologically possible deviance. One way of solving this problem would be Malmgren's possibility claim, modified as described above.

(v) The problem of missing the target is mitigated as far as nomological necessity as a fallback position of the $J T B$-theory is blocked. At the same time, there is an easy road to metaphysical modality. Assuming that nomological possibilities form a subset of metaphysical ones, if a scenario is nomologically possible, it is metaphysically possible. And if the JTB-theory is not true for all nomologically possible scenarios, a fortiori it is not true for all metaphysically possible scenarios. However, weaker fallback positions like a claim about past, present and future cases of knowledge remain.

In sum, the general strategy of restricting the realm of alethic possibilities is promising if

9 I have argued that, in Gettier's times, metaphysical modalities were more contentious than they are today. For instance, Seddon (1972) runs together logical with nomological possibility and positive probability, i.e. epistemic possibility. Perhaps Gettier held a similar view. 
one is reluctant to reason in terms of metaphysical modality, but some metaphysical concerns remain, and there are additional epistemic requirements like knowing the laws. Moreover, the strategy clearly does not work for philosophical scenarios which are agreed to go beyond the nomologically possible. Notwithstanding these limitations, it seems worthwile to write a suitable version of Option I into the disjunction of alternative formalisations of a thought experiment like Gettier's

In the next sections, I shall discuss a more radical alternative: instead of alethic modality, I use epistemic modality.

\subsection{Option II: Epistemic Possibilities}

The most literal interpretation of Gettier's argument is that he introduces a supposition as expressible by an indicative conditional. I shall pursue this literal interpretation. I cannot dig too far into the huge debate on indicative conditionals. One useful platitude to start with is that counterfactuals deal with metaphysical possibilities, whereas indicative conditionals deal with epistemic possibilities. I shall try to make do with this idea and a rough understanding of epistemic possibility: $p$ is epistemically possible relative to some subject $S$ just in case $p$ is compatible with $S$ 's knowledge. Instead of $S$ 's knowledge, one may consider what is presupposed as known in a context $C$. Besides being faithful to Gettier's words, the main advantage of using epistemic possibilities is that they are metaphysically innocent. They are grounded in our limited knowledge of the world. ${ }^{10}$ I shall discuss two exemplary ways of using epistemic possibilities: a priori credences and current epistemic possibilities. Each comes with its own advantages and disadvantages. Together they constitute a set of highly relevant alternatives to reasoning by alethic

10 Some philosophers construe epistemic possibilities in terms of centred worlds (e.g. Chalmers 2011). Thus, it is not fully clear that epistemic possibilities evade the ideological complexities of a possible worlds-framework. 
possibilities.

\subsubsection{First Variant: A Priori Knowledge}

I shall start with an epistemic category which has traditionally been associated with metaphysical modality, but may also be used to replace it: Rachael Briggs has proposed one reading of the necessity operator as 'it is knowable a priori that...'(2009, 433). ${ }^{11}$ There are other readings, of course, which yield a posteriori necessities.

Presenting the target theory (JTB-theory) as a claim to a priori knowledge will seem objectionable to philosophers who doubt the significance of the a priori/a posteriori distinction. But this apparent disadvantage may be owed to the very bias in favour of metaphysical modalities which I want to challenge. If the bias is challenged, we are faced with the choice between two different readings of the modal operator. Both may come with their own problems. There are even philosophers who insist on the aprioricity of the Gettier argument (Ichikawa and Jarvis 2009, 2013, Malmgren 2011). But aprioricity has not yet been considered as an alternative to metaphysical modality. Depending on how a priori is understood, my proposal covers concept-based and rationalist views of thought experiments. According to the former, Gettier's argument is a piece of purely conceptual reasoning (Ichikawa and Jarvis 2009, 2013). According to the latter, our verdict on the case is a rational intuition (Malmgren 2011). In turn, these approaches lend themselves to answering epistemological qualms about a priori knowledge.

Thus, as a first stab, I shall consider a reading of 'necessarily' as 'it is a priori knowable that...' and of 'possibly' as 'it is not a priori knowable that not...'. I shall argue, however, that (perhaps unsurprisingly) this reading does not completely evade the problems with metaphysical modalities. Then I shall explore measures to evade them.

The first two premises of the a priori reading are:

11 The original context of Briggs's proposal is formulating Lewis's Principal Principle. 
It is a priori knowable that, for any subject $S$ and proposition $p, S$ knows $p$ if and only if $S$ has justified true belief in $p$.

It is not a priori knowable that it is not the case that some $S$ is in $G C$.

However, the cornerstone of any reading is the conditional premiss. Conditionals are notoriously difficult to understand, and so is their role in the argument. A natural starting point is a material conditional: someone is in $G C \supset$ Someone has $N K J T B$. But this conditional is vulnerable to the problem of deviant realisations (iv). It is likely to be false if $G C$ happens to be realised deviantly. Instead of a material conditional, we might try some stronger reading: many philosophers treat an indicative conditional if $p$ is the case, $q$ is the case not merely as a material conditional but analyse it by a necessity operator scoped over a material conditional (Williams 2008, Chalmers 2011, Ichikawa 2011, Rothschild 2013). Since the necessity operator is interpreted as context-dependent, this approach is more flexible than a material conditional. One may exclude deviant realisations even if they are actual. In my adaption, the possibilities the necessity operator ranges over are epistemic possibilities. Interpreting the conditional premiss along these lines yields the following: all relevant epistemic possibilities where someone is in $G C$ are possibilities where someone has NKJTB. But what are the relevant epistemic possibilities? In line with the a priori reading, we have to say: those not ruled out by what we know a priori. This answer enmeshes us in the problem of deviant realisations. It is not a priori excluded that the Gettier scenario is realised in a deviant way. The conditional amounts to a false claim: all realisations of $G C$ that are not ruled out by what we know a priori are cases of $N K J T B$.

I shall very briefly discuss four ways of evading the problem. The first again is inspired by Malmgren (2011). Abandon the conditional premiss and merge it with the second premiss into a conjunctive possibility claim: 
It is not a priori excluded that someone in $G C$ has $N K J T B$.

I have already voiced my concern that the connection between $G C$ and NKJTB becomes too tenuous. In 2.2.2., I shall present a weakened material conditional (Alternative $D, \mathrm{D} 3$ ) where the connection between $G C$ and $N K J T B$ is comparably tenuous, mainly because (D3) is easier to know than stronger rivals. Here I shall try to build a stronger premiss.

The second attempt at evading the problem of deviant realisations is to read the Gettier description as a piece of fiction (Ichikawa and Jarvis 2009, 2013). In evaluating a fiction, we go beyond the explicit story. We seem to dismiss deviant realisations of the story. This feature of fictions may be used to close in onto the non-deviant possible realisations of the scenario. I do not deny that thought experiments ultimately may involve or even be fictions, especially in a context of discovery. Gettier himself used fictional proper names. But when building a formalisation, we cannot simply rely on fiction to be suitably well-behaved to yield the right possible situations. For instance, one may write a story where someone has refuted Gödel's Theorem (Currie 1990, 87). One may even write a story where this is not explicitly said but an implicit fictional truth. Perhaps fictions in philosophical contexts are more regulated (Meynell 2014). But we would need an argument why we are allowed to assume this.

The third attempt at evading deviant realisations is to claim that the context of relevant scenarios shrinks on the way to the conditional premiss. The first premiss requires us to take into account all epistemic possibilities that are not a priori excluded. Some later premiss requires us only to take into account epistemic possibilities which, given our current knowledge, cannot be ruled out or appear too outlandish. This shift in context seems ad hoc, though. It is often assumed that the range of contextually salient possibilities is easily expanded but not so easily contracted (Gillies 2007). As I shall argue, we are ready to accommodate even far-fetched epistemic possibilities when they are somehow made salient. But we are not as ready to disregard these possibilities once they are on the table. Thus, it seems ill-advised to claim that the second or third premiss contracts the 
range of relevant epistemic possibilities.

A fourth way of evading the problem is to reinterpret the conditional premiss as follows: Briggs characterises being knowable a priori as deserving maximum rational credence 1 (on a scale between 0 and 1). Rational credence 1 for some $p$ (and 0 for not- $p$ ) cannot be changed by any evidence. One may interpret a conditional if $p$ is the case, $q$ is the case according to Adam's thesis: rational credence in the conditional equals rational credence in $q$ given $p$. The thought experiment can be construed accordingly. Let $\operatorname{Cr}(q)$ be the current rational degree of credence in some $q$, $\operatorname{Cr}(q / E)$ rational credence in $q$ conditional on evidence $E$ (e.g. evidence that some $S$ is in $G C$ ):

Alternative $C$

(C1) JTB-theory: $C r$ (for any subject $S$ and proposition $p, S$ knows $p$ if and only if $S$ has justified true belief in $p)=1$

From $(\mathrm{C} 1)$

(C2) For any $E, C r$ (for any $S, p, S$ knows $p$ if and only if $S$ has justified true belief in $p / E)=1$

From (C2)

(C3) For any $E, C r($ some $S$ has justified true belief in some $p$ but does not know $p / E)=0$

(C4) It is not the case that $C r$ (some $S$ is in $G C)=0$

(C5) It is not the case that $C r$ (some $S$ has justified true belief in some $p$ but does not know $p /$ Some $S$ is in $G C)=0$

Thus: not (C1)

Alternative $C$ evades the problems (i) and (iv) from section 1. Concerning (iii), Gettier does not mention aprioricity, but we get a neat reading of his 'suppose' + indicative. As for problem (ii), epistemological challenges of knowing metaphysical possibilities are traded against challenges of a priori knowledge. I dare not decide whether this is an advantage, but at least we are given the choice. There are several possibilities for an epistemology of the a priori. One is a priori 
conceivability (Chalmers 2002), but this may lead us back to something very close to the epistemology of metaphysical modality. Another possibility is to carefully spell out epistemological requirements for a capacity of rational intuition (cf. Bengson 2015). I shall briefly lay out a third alternative: A priori knowledge is characterised by conceptual knowledge. As for (C4), for us to assign zero credence to $G C$, purely conceptual knowledge should allow to rule out $G C$, but it doesn't. Thus, we assign some positive credence, however minimal, to GC whatever evidence is coming in. ${ }^{12}$ As for (C5), purely conceptual knowledge together with the assumption that there is a case of $G C$ does not rule out that there is a case of NKJTB. As a consequence, we should assign positive credence to there being a case of $N K J T B .{ }^{13}$ This sketch provides an exemplary illustration of epistemological commitments of a priori knowledge. Notwithstanding these substantial commitments, Alternative $C$ provides an epistemologically interesting alternative to knowledge of metaphysical modality.

However, Alternative $C$ does not evade problem (v). It narrows down the dialectical potential of $G C$. The argument may refute the strong claim that the $J T B$-theory deserves credence 1 . But what about the weaker claim that current rational credence in the JTB-theory is lower than 1 but still reasonably high, high enough for the theory to count as knowledge? Still Alternative $C$ is an interesting alternative to Alternative $A$. We trade one narrowing problem against another. And committing oneself only to the disjunction of Alternatives $A, B, C$ reduces the available countermoves to save the $J T B$-theory.

Another limitation is that the a priori template cannot be extended to thought experiments both in philosophy and science which recruit empirical knowledge over and above a priori 12 As a consequence of conceiving of aprioricity in terms of credence 1 and 0 , respectively, no empirical evidence can yield absolute certainty that there never is a case of $G C$ (which is NKJTB). 13 One might object that (C5) in this view does not reflect the positive evidential role of $G C$. But if the thought experiment is construed as an exercise of conceptual knowledge, it is to be expected that $G C$ mainly plays the psychological role of activating implicit conceptual knowledge. 
knowledge.

While I reckon Alternative $C$ a good candidate for being written into the disjunction of formalisations the thought experimenter ought to commit herself to, its limitations motivate looking for a further alternative which makes do with the weakest version of the $J T B$-theory.

\subsubsection{Second Variant: Knowledge given the Present Context}

The doubts just outlined motivate to consider an alternative understanding of Gettier's 'suppose'. It avoids the narrowing problem by abandoning the claim to a priori knowledge, starting from epistemic possibility given what we presently know. Instead of an a priori claim, the $J T B$-theory is put as a simple biconditional: one has knowledge precisely if one has justified true belief. The JTBtheory surely comes with a claim to knowledge. Knowing that knowledge is JTB seems to require that the contextually relevant alternatives can be ruled out (Lewis 1996). So the target claim becomes:

In all epistemic possibilities (in the current context $C$ ), for all $S, p, S$ has knowledge of $p$ if and only if $S$ has justified true belief in $p$.

Accordingly, the second premiss becomes:

There is an epistemic possibility (in $C$ ) that some $S$ is in $G C$.

As for the third premiss, the epistemically least demanding way of putting it is the following material conditional:

There is an epistemic possibility (in $C$ ) that some $S$ is in $G C \supset$ There is an epistemic possibility (in 
$C$ ) that some $S$ has justified true belief in some $p$ without knowing $p$.

It does not matter whether a deviant realisation of $G C$ is actual because we only need that a normal case of $G C$ is epistemically possible. Considering my misgivings about Malmgren's (2011) a priori possibility claim in section 2.2.1., one may feel that the connection between $G C$ and $N K J T B$ in this material conditional is just as tenuous. But firstly, it is natural to read the conditional as part of a modus ponens argument from $G C$ to $N K J T B$. Secondly, as distinguished from Malmgren's a priori premiss or my a priori Alternative $C$, all empirical information about actual justification and knowledge can be used to build connections between $G C$ and NKJTB. If the relationship still appears too tenuous, a way of strengthening the conditional premiss would be a reading where all contextual $G C$-possibilities are $N K J T B$-possibilities. ${ }^{14}$ In my exemplary formalisation, I refrain from using this stronger premiss as it is epistemically more demanding.

The full argument becomes:

Alternative $D$

(D1) In all epistemic possibilities (in the current context $C$ ), for all $S, p, S$ knows $p$ if and only if $S$ has justified true belief in $p$.

(D2) There is an epistemic possibility (in $C$ ) that some $S$ is in $G C$.

(D3) There is an epistemic possibility (in $C$ ) that some $S$ is in $G C \supset$ There is an epistemic possibility (in $C$ ) that some $S$ has justified true belief in some $p$ without knowing $p$.

(D4) There is an epistemic possibility (in $C$ ) that some $S$ has justified true belief in some $p$ without knowing $p$.

Thus: not (D1)

14 For the stronger premiss to work, deviant realisations would have to be dismissed as too outlandish in the current context (cf. Williams 2008, 216). 
How do we figure out (D2) and (D3)? As for (D2), while there is a lot of debate on epistemic modals, access to epistemic possibilities in one's present context tends to be taken for granted. This may be explained as follows: outright beliefs are mostly accessible. Unless one is in the grip of radical scepticism, it seems plausible that our beliefs in turn sufficiently often amount to knowledge to provide reliable guidance to what is consistent with current knowledge. Thus, access to our beliefs also provides access to what is epistemically possible in our current epistemic situation.

I present an exemplary way how to figure out the conditional premiss (D3): given (D2), it is natural to read the antecedent as part of modus ponens reasoning to the consequent. The epistemic possibility of $G C$ in (D3) is read as positively bearing on the epistemic possibility of $N K J T B$. The easiest way for the former to bear on the latter is to consider $G C$ as a case of NKJTB. When assessing the epistemic possibility of $G C$, we are aware of some most readily accessible $G C$ possibilities, at least to some degree of specification. Even if one happens to know that some deviant realisation of $G C$ is actual, normal realisations will be among those which most readily come to mind. ${ }^{15}$ There is a capacity to classify such epistemic possibilities with respect to justification and knowledge. This reliable, though defeasible capacity is acquired mainly by classifying actual cases. We treat the most salient epistemic possibilities like corresponding actual cases. Yet there is one important difference. The actual cases we encounter are fully concrete, although information about them is limited. In contrast, the possibilities within our purview are incomplete although they could be further specified. Even if they so far do not seem deviant, they normally will cover concrete subcases which are NKJTB and concrete subcases which are not. Still we find it likely for any subcase that it will be $N K J T B$, although we might be unable to specify probabilities. Hence we accept that there is a significant possibility of some $G C$-possibility being a case of NKJTB. This is sufficient to support (D3).

I cannot go into the full details of the classificatory capacity involved. One prominent

15 The cognitive mechanism guiding this awareness may be availability heuristics (on the epistemological significance of the latter cf. Hawthorne 2004, Williamson 2005). 
proposal in the literature is to describe it as mindreading heuristics (Nagel 2012). I am neutral with respect to this proposal, but it applies at best to a limited range of thought experiments. A more general alternative might be the use of representativeness heuristics (cf. Nilsson et al. 2008): to check whether a case description falls under a category, it is compared to representative instances of the category stored in memory. If the limited description is sufficiently similar to these instances along relevant dimensions, it is defeasibly classified as falling under the category. When we consider a normal stopped-clock case $G C$, it compares well to representative instances of epistemic justification, looking at a clock being an established method of figuring out the time. It does not matter that the clock is broken and just happens to display the right time. However, taking into account these features, the case does not compare sufficiently well to representative instances of knowing but rather to instances where something goes wrong with acquiring knowledge (inadvertently looking at a malfunctioning measurement device). Hence the tendency to classify $G C$ as NKJTB. Given the biases of representativeness heuristics, I intend these remarks only as a tentative suggestion subject to further research. What is decisive is that the capacity of epistemic classification is on the whole reliable.

Postponing principled worries about using epistemic possibilities to the final section, I shall discuss some controversial aspects of Alternative $D$ in particular:

Use of epistemic possibilities seems to come with an undue relativity to our epistemic situation. What is compatible with our knowledge is subject to constant changes. The same goes for our credence in $G C$. Sometimes we may be able to rule out altogether that anyone is in $G C$. But it would seem preposterous to assume that the persuasiveness of the Gettier experiment depends on our changing information about $G C$ being actual.

The formalisations considered differ in their sensitivity to one's actual epistemic situation. The first variant (Alternative $C$ ) is couched in terms of a priori knowledge. It fixes the relevant epistemic situation to minimal empirical information and hence does not depend on changes of this epistemic situation. The second variant (Alternative $D$ ) is sensitive to our actual epistemic situation. 
It is not sensitive to how much credence precisely is assigned to $G C$, but if the thought experimenter is in a position to empirically exclude that $G C$ is ever actual, for instance in a universe with a few people which is about to end, Alternative $D$ is of no avail. Alternative $D$ depends on the contextual epistemic possibility of $G C$, but the thought experiment does not. This difference surely reduces the plausibility of Alternative $D$.

There are some considerations to allay this concern. We are willing to make room even for slight epistemic possibilities for the sake of argument. Alternative $D$ might be used unless we definitely rule out the epistemic possibility of $G C$. Moreover, Alternative $D$ forms part of a bundle of alternatives, which may replace it. When a case is introduced by 'suppose' and 'imagine' + indicative mood, it is natural to first think of an epistemic possibility given our current situation. In the unlikely event that the epistemic possibility is definitely ruled out, there is the alternative of retreating to aprioricity or alethic possibility. Due to such fallback options, we feel that the thought experiment does not depend on current epistemic possibilities. But it does not follow that current epistemic possibilities are not the option to begin with. The fallback structure seems complicated when we attend to it, but in any normal situation only the most immediate option is in focus.

In Alternative $D$, the target analysis of knowledge is put as a claim to knowledge. This would be problematic if either there were a circle in the definition, or a specific notion of knowledge were presupposed. But there is no circle. Knowledge does not figure in the necessary and sufficient conditions of knowledge. Moreover, no particular analysis of knowledge is presupposed. It is assumed that knowledge requires to rule out any contextually salient alternative. But this presupposition is not too much of a burden.

There are doubts about representing the $J T B$-theory as ruling out epistemic possibilities. It is not the same to refute the claim that knowledge is $J T B$ and the claim that one knows this. Refuting the latter claim is compatible with knowledge being $J T B$, i.e. the $J T B$-theory being true. So the counterexample would not show that the JTB-theory is false! This adds to the doubts that Alternative $D$ is sufficiently faithful to Gettier's original presentation. Gettier surely took his 
example to directly establish that the $J T B$-theory is false. Still there is a very close connection between refuting a claim to knowledge and rebutting what is claimed to be known. The only way of saving the $J T B$-theory from the counterexample would be to weaken its purported epistemic status to something short of knowledge but still bearing on the epistemological key question: what is knowledge? But since the relevance of this question mainly depends on knowledge being the standard of epistemic success, it would seem a desperate move for the JTB-theorist to retreat to a claim that falls short of knowledge.

Notwithstanding the concerns outlined, I deem Alternative $D$ relevant to spelling out Gettier's heuristic. I shall point out some important advantages:

As I have argued, Alternative $D$ tracks the dialectical potential of Gettier's example. While the proposal evades the problem (v) of weakening the potential of the counterexample, in turn there are ways of extending it such as to arrive at stronger claims. If it cannot be ruled out that there is a case of NKJTB given all our present knowledge, it cannot be ruled out a priori either. Thus, there is a direct transition from the argument to an a priori version. There is also a transition to metaphysical modalities: what is actual is possible. Hence the epistemic possibility of someone having NKJTB entails the epistemic possibility of someone possibly (metaphysical possibility) having NKJTB and, given a fixed context, is incompatible with knowing that, necessarily, any case of justified true belief is a case of knowledge.

Weighing the advantages and disadvantages of Alternative $D$, I propose it as a further candidate for the disjunction of ways to make the Gettier experiment precise. I have distinguished two exemplary ways of formalising the Gettier experiment as an epistemic possibility: the target claim may be represented as knowable a priori and as known simpliciter (given the current context). Both somewhat depart from Gettier's explicit presentation. Still, they preserve a lot of the immediate appeal of Gettier's original reasoning. We start from a claim to knowledge, accept the Gettier story as something that might well happen, and use a corresponding supposition to proceed by an everyday indicative conditional to the consequence that conflicts with the target claim. 


\section{Do Metaphysical Modalities Strike the Best Balance?}

I have presented readings of the Gettier experiment which avoid any commitment to metaphysical modalities. Moreover, they are serious competitors both for the most natural reaction to Gettier's actual text and for a standard formalisation of refuting thought experiments. However, there is a suspicion that the Gettier experiment can do without metaphysical possibilities precisely because it features such a down-to-earth scenario. There are other scenarios which require to dwell on metaphysical modalities, one may say. And so metaphysical modalities come for free in the Gettier case. Indeed metaphysical modalities and metaphysically possible worlds may eventually turn out to constitute the best unificatory framework for thought experiments and other kinds of more or less explicitly modal reasoning. Yet even if metaphysical modalities are preferable upon ideal philosophical reflection, it does not follow that they figure in a construal of Gettier cases and the like which, taken in isolation, comes with a minimum of problematic commitments. So far my aim has been to outline alternative candidates for such a construal. It is a matter of further debate whether we ultimately should settle for metaphysical modalities instead. In this section, I shall further explore the boundaries of my strategy.

I have already noted that my Alternative $B$ is unavailable for counternomological thought experiments. Epistemic possibilities are more flexible. Still epistemically far-fetched thought experiments may provoke resistance. Besides the general flexibility of my disjunctive strategy, there are two more specific reactions to this concern.

One option is to bite the bullet and insist that this is why far-fetched thought experiments are problematic. Just as there is a concern that far-fetched thought experiments invoke too eccentric metaphysical possibilities, there may also be a concern that they invoke too far-fetched epistemic possibilities to be taken seriously. However, being epistemically and being metaphysically farfetched do not always go together. For instance, Nozick's scenario of living in a pleasure machine 
seems epistemically but not metaphysically far-fetched. There is no pleasure machine, but there could be one. Thus, we may dismiss a thought experiment because it is epistemically far-fetched although the relevant question would be whether it is metaphysically far-fetched.

The alternative to just biting the bullet is to point to our willingness to make room for scenarios for the sake of argument, even when we are in a position to rule them out. For instance, Stephen Yablo (2002) accepts the indicative conditional

If 'tail' means 'wing', horses do not have tails.

Although we know that 'tail' does not mean 'wing', we nevertheless make room for the epistemic possibility that it does. The same might go for far-fetched thought experimental situations like zombies or Avicenna's floating man. It is not only perfectly all right to treat Gettier cases as epistemic possibilities, the same should even go for more far-fetched thought experiments, at least in a philosophical discussion. We may be willing to take seriously very slight epistemic possibilities given the current context. But sometimes it may be advisable to use a priori epistemic possibilities as in Alternative $C$, which go far beyond epistemic possibilities given our present epistemic status.

So far I have considered the concern that epistemic possibilities might be too restrictive. But there is also the opposite concern. One may rationally acknowledge a scenario as epistemically possible, say that Goldbach's Conjecture is false, without thereby accepting its metaphysical possibility. Hence an epistemic construal might lead to accepting counterexamples which are epistemically but not metaphysically possible. Still such counterexamples may play their role in shaking belief in a target theory. Moreover, any salient epistemic possibility will be a candidate for being actual and thus for being a metaphysical possibility. I admit that thought experiments which aim at establishing a metaphysical possibility may need more than just epistemic possibility. But for such thought experiments, modesty in modal commitments is not an option anyway.

A related concern about the Gettier experiment: under certain assumptions, even a case of 
NKJTB may be epistemically but not metaphysically possible. For instance, one may assume that 'knowledge' behaves in certain respects like natural kind terms. As a consequence, the deep structure of knowledge goes beyond our everyday concept (Weatherson 2003, Williamson 2007, 206). ${ }^{16}$ It is revealed by a theory that balances intuitions against theoretical virtues like simplicity, fruitfulness etc.. Contrary to our intuitions, the $J T B$-theory might strike the best balance. Then the Gettier story as an instance of $N K J T B$ is now an epistemic but not a metaphysical possibility. As a consequence, Gettier's argument put in terms of epistemic possibilities does not rule out that knowledge necessarily is JTB. In my view, the concern just reveals a potential limit of counterexamples like Gettier's, regardless of whether we treat them as epistemic or metaphysical possibilities. There might be a hidden deep structure which is missed by our intuitions.

I admit that it often makes a difference whether a case is treated as an epistemic or as a metaphysical possibility, especially in epistemological debates. I shall consider two cases which seem to require reasoning in terms of metaphysical modalities, Twin-Earth experiments and sceptical scenarios:

A kind of thought experiment which had an impact similar to Gettier's are Putnam- and Kripke-style examples which show that meanings are not in the head, certain expressions are used as rigid designators, informative identity statements like 'Hesperus is Phosphorus' are necessary albeit a posteriori and so on. One lesson that has been drawn from this kind of thought experiment is to distinguish scenarios imagined as actual and as counterfactual (Chalmers 2002). However, the most important results may well be attained without dwelling on counterfactuals. Putnam's original argument for meaning externalism has not been put in terms of the metaphysical possibility of a twin earth but as a science fiction story about a mission to a distant planet (Putnam 1990, 70). We may accordingly distinguish several dimensions of meaning, depending on the epistemic possibility considered. Suppose there is a distant planet where $X Y Z$ plays the role water plays on earth; still the

${ }_{16}$ We are still far from the claim that epistemology should be replaced by empirical science (cf. Pernu 2009). 
$X Y Z$ on that planet is not water. But suppose the stuff that plays the water role on earth turns out to be $X Y Z$. Then arguably water is $X Y Z .{ }^{17}$ Thus, we can learn a lot about how we use terms like 'water' without dwelling on counterfactuals or metaphysical modalities. ${ }^{18}$ What cannot be attained are claims which are put as outright metaphysical modalities, such as 'water is necessarily $\mathrm{H}_{2} \mathrm{O}$ ' or "'water" is a rigid designator'. Such claims seem to stand and fall with accepting metaphysical modalities.

Coming to sceptical scenarios, surely it makes a difference to reason what would be the case if we were brains in vats and what is the case if we are brains in vats. It would be surprising if the difference were not relevant to thought experimenting. Another kind of scenario even better illustrates the point: scenarios featuring one's own non-existence. For instance, I have difficulties to make room for the supposition that I do not presently exist. Perhaps I can consider this supposition, but it probably will impose very different requirements and lead to very different results than considering what would be the case if I did not exist. Again counterfactuals may prove unavoidable, raising issues whether they come with a commitment to metaphysically possible worlds. One option for representing such scenarios are nomological possibilities as used in my Alternative $B$.

Yet even if we eventually have to settle for metaphysical possibilities, as long as we are concerned only with Gettier experiments and their kin, we may hold onto the alternatives I have sketched as a way to minimise our commitments and to maximise breadth of application. My proposal is to supplement Gettier's heuristic reasoning by committing oneself only to a disjunction of precisifications, some of them featuring alethic (nomological, metaphysical...) and some of them featuring epistemic possibilities, without committing oneself to one of the precisifications in ${ }_{17}$ To put it more cautiously: the proposition I express by the string of words 'water is $X Y Z$ ' is true, and it differs from the one I express by the same string given water is $\mathrm{H}_{2} \mathrm{O}$.

18 A case that illustrates the advantages of not simply going for possible worlds is the debate on the relevance of slow-switching arguments, issuing in the real-life chicory-arugula example (Ludlow 1995). 
particular. More-generally, I suggest that thought experimenters be sensitive to their situationspecific minimum commitments instead of heading for metaphysical modality without further ado.

Bengson, J. 2015. The Intellectual Given. Mind 124, 707-760.

Briggs R. 2009. The Anatomy of the Big Bad Bug. Noûs 43, 428-449.

Chalmers, D. 2002. Does Conceivability Entail Possibility? In Conceivability and Possibility, ed. T. S. Gendler \& J. Hawthorne, 71-125. Oxford: Clarendon Press.

Chalmers, D. 2011. Frege's Puzzle and the Objects of Credence. Mind 120, 587-635.

Currie, G. 1990. The Nature of Fiction. Cambridge: Cambridge University Press.

Geirsson, H. 2005. Conceivability and Defeasible Modal Justification. Philosophical Studies 122, 279-304.

Gettier, E. 1963. Is Justified True Belief Knowledge?. Analysis 23, 121-123.

Gillies, A. S. 2007. Counterfactual Scorekeeping. Linguistics and Philosophy 30, 329-60.

Grundmann, T., Horvath, J. 2014. Thought Experiments and Deviant Realizations. Philosophical Studies 170, 525-533.

Hawke, P. 2011. Van Inwagen's Modal Scepticism. Philosophical Studies 153, 351-364. 
Hawthorne, J. 2004. Knowledge and Lotteries. Oxford: Oxford University Press.

Horvath, J. 2015. Thought Experiments and Experimental Philosophy. In The Palgrave Handbook of Philosophical Methods, ed. C. Daly, 386-418. Basingstoke: Palgrave Macmillan.

Huemer, M. 2009. When is Parsimony a Virtue?. The Philosophical Quarterly 59, 216-36.

Ichikawa, J. 2011. Quantifiers, Knowledge, and Counterfactuals. Philosophy and Phenomenological Research 82, 287-313.

Ichikawa, J., Jarvis, B. 2009. Thought Experiment Intuitions and Truth in Fiction. Philosophical Studies 142, 221-246.

Ichikawa, J., Jarvis, B. 2013. The Rules of Thought. Oxford: Oxford University Press.

van Inwagen, P. 1998. Modal Epistemology. Philosophical Studies 92, 67-84.

Langland-Hassan, P. 2014. Imaginative Attitudes. Philosophy and Phenomenological Research 88, 664-686.

Lewis, D. 1986a. On The Plurality of Worlds, Oxford: Blackwell.

Lewis, D. 1986b. Counterfactual Dependence and Time's Arrow, Postscripts to 'Counterfactual Dependence and Times's Arrow'. In Philosophical Papers: Volume II, 32-66. Oxford: Oxford University Press. 
Lewis, D. 1996. Elusive Knowledge. Australasian Journal of Philosophy 74, 549-67.

Lowe, E.M. 2012. What Is the Source of Our Knowledge of Modal Truths?. Mind 121, 919-950.

Ludlow, P. 1995. Externalism, Self-Knowledge, and the Prevalence of Slow-Switching. Analysis 55, 285-286.

Malmgren, A.S. 2011. Rationalism and the Content of Intuitive Judgements. Mind 120, 263-327.

Meynell, L. 2014. Imagination and Insight. A New Account of the Content of Thought Experiments. Synthese 191, 4149-4168.

Nagel, J. 2012. Intuitions and Experiments. Philosophy and Phenomenological Research 85, 495527.

Nilsson, H. et al. 2008. Exemplars in the Mist. The Cognitive Substrate of the Representativeness Heuristics. Scandinavian Journal of Psychology 49, 201-212.

O’Leary-Hawthorne, J. 1996. The Epistemology of Possible Worlds: A Guided Tour. Philosophical Studies 84, 183-202.

Pernu, T. 2009. Is Knowledge a Natural Kind?. Philosophical Studies 142, 371-386.

Putnam, H. 1990. Realism with a Human Face. Harvard: Harvard University Press. 
Quine, W. V. O., 1953. Three Grades of Modal Involvement. Proceedings of the XIth International Congress of Philosophy 14, 65-81.

Roca-Royes, S. 2011. Conceivability and De Re Modal Knowledge. Noûs 45, 22-49.

Rosen, G. 1993. A Problem for Fictionalism about Possible Worlds. Analysis 53, 71-81.

Rothschild, D. 2013. Do Indicative Conditionals Express Propositions?. Noûs 47, 49-68.

Seddon, G. 1972. Logical Possibility. Mind 81, 484-494.

Smith, M. 2007. Ceteris Paribus Conditionals and Comparative Normalcy. Journal of Philosophical Logic 36, 97-121.

Weatherson 2003. What Good Are Counterexamples?. Philosophical Studies 115, 1-31.

Williams, J.R.G. 2008. Conversation and Conditionals. Philosophical Studies 138, 211-23.

Williamson, T. 2005. Contextualism, Subject-Sensitive Invariantism and Knowledge of Knowledge. Philosophical Quarterly 55, 213-35.

Williamson, T. 2007. The Philosophy of Philosophy. Oxford: Blackwell.

Williamson, T. 2009. Replies to Ichikawa, Martin and Weinberg. Philosophical Studies 145, 465- 
476.

Yablo, S. 1993. Is Conceivability a Guide to Possibility?. Philosophy and Phenomenological Research 53, 1-42.

Yablo, S. 2002. Coulda, Woulda, Shoulda. In Conceivability and Possibility, ed. T. Gendler and J. Hawthorne, 441-492. Oxford: Clarendon Press. 